\title{
Los catecúmenos de la aceleración. Deporte de alto rendimiento y vértigo social
}

\author{
The catechumens of acceleration:
}

High-performance sport and social vertigo

\section{Agustín Aranco Bagnasco*}

\footnotetext{
* Maestrando en Ciencias Humanas (opción Filosofía Contemporánea) y licenciado en Filosofía por la Facultad de Humanidades y Ciencias de la Educación, Universidad de la República, Uruguay. Docente en la misma universidad. -agustinarancob@gmail.com http://orcid.org/0000-00019553-6825
}

\section{Resumen}

Por detrás de la presuntamente intuitiva relación entre el deporte y la sociedad, coexisten diferentes maneras de analizar la normatividad compartida por ambas esferas. En lo que respecta al deporte de alto rendimiento, como extensión y ampliación histórica del deporte moderno, no se han producido desarrollos concluyentes que recojan uno de los diagnósticos tardomodernos más sugerentes y explicativamente satisfactorios: el de la aceleración social. Este artículo se sirve de las herramientas de la teoría y la filosofía social, así como, en menor medida, de las categorías de la filosofía del deporte contemporáneo, para indagar sobre la conexión entre la forma de vida contemporánea y los principios, reglas y valores que dominan la generalidad del deporte de alto rendimiento en la actualidad, que se concreta en fenómenos como el dopaje. Se argumenta que esta conexión constituye una línea de análisis plausible para superar las habituales discusiones y diatribas entre externalismo e internalismo, puesto que permite poner de relieve determinaciones culturales que, al ser moralmente indeseables e indeseadamente promovidas por quienes consagran su tiempo al fenómeno deportivo (como espectadores o consumidores), logran explicar la funcionalidad que cumple el deporte de alto rendimiento en la reproducción de cierto marco socioontológico general dentro de las sociedades contemporáneas.

Palabras clave: deporte, sociología del tiempo libre, atleta. 


\section{Abstract}

Behind the allegedly intuitive relationship between sport and society, different ways of analyzing the normativity shared by both spheres coexist. Regarding high-performance sports, as a natural extension of modern sport, there are non conclusive developments comprising one of the most suggestive and explanatory late-modern diagnoses: that of social acceleration. Consequently, the article investigates the connection between the contemporary way of life and the principles, rules and values that dominate the generality of high-performance sports today, by means of theory and social philosophy tools, and to a lesser extent, of contemporary philosophy-of-sport's categories. It is argued that this connection constitutes a plausible line of analysis to overcome the usual discussions and diatribes between externalism and internalism, since it allows to highlight cultural determinations that, being morally undesirable and unwantedly promoted by those who dedicate their time to the phenomenon of sport (as spectators or consumers), manage to explain the functionality that high-performance sports fulfills in the reproduction of a certain general socio-ontological framework within contemporary societies.

Keywords: philosophy of sport, sociology of leisure time, athlete.

\section{Introducción}

En mayo de 1916, Filipo Tomasso Marinetti publica un brevísimo manifiesto futurista en el que reivindica, en línea con la tónica de su prédica, la civilización moderna, la vertiginosidad de los vehículos, al tiempo que observa en la tradición cristiana un lastre incómodo frente a la tarea expansiva y escalatoria en la que creía ver la oportunidad de apropiarse definitivamente del tiempo y el espacio (Marinetti, 2009). Quien fuera uno de los principales impulsores del movimiento estético-político de comienzos de siglo XX italiano, y hasta servidor intelectual en la tarea de cementar varias de las ideas que hicieron posible el ascenso al poder de Mussolini (Ialongo, 2013), caracterizaba su novel planteo como la «religión-moralidad» de la velocidad y el vértigo social (Marinetti, 2009). ${ }^{1}$ Lo importante de todo esto es que, hacia el final del mencionado manifiesto, Marinetti se refiere a los deportistas (sportmen) como los «catecúmenos» (2009, p. 59), que, en calidad de auténticos ejemplares de lo que se estaba propugnando, debían ser los primeros en recibir la bendición. La brevedad y la concisión del manifiesto no nos permite ahondar en las razones de esta analogía trazada por Marinetti, lo que no impide, en ningún caso, que podamos elaborar una hipótesis acerca de la proximidad normativa 
entre el deporte de alto rendimiento y algunos rasgos de la radicalización de los valores modernos que tiene lugar, al menos, desde las últimas dos décadas del siglo XX. Según lo veremos en lo que resta del artículo, ambos parecen articularse, ciertamente, alrededor de un ideal, y en especial de una promesa, que surge junto con la vía secularizada de la aceleración social (Rosa, 2016).

La recepción de la estética futurista en Uruguay durante el ocaso decimonónico, al menos en lo que respecta a la narrativa y la poesía, halló su lugar en la obra de dos figuras relativamente eclipsadas en la historia de la cultura literaria nacional, a saber, Alfredo Mario Ferreiro (1899-1959) y Juan Parra del Riego (1894-1925). Este último, sin ir más lejos, en una oda a Isabelino Gradín, jugador de la selección uruguaya de fútbol y representante del país en las distintas versiones de la Copa América de la primera década del siglo XX, no escatimaba en elogios a la velocidad del delantero. Como el poema que abre sus Polirritmos, y en medio de otros versos dedicados al enaltecimiento de los motores (especialmente al de la motocicleta) y el elogio de la movilidad y el desplazamiento continuo que caracterizan a la urbanidad, Parra del Riego lo expresaba en los siguientes términos: «Palpitante y jubiloso / como el grito que se lanza de repente a un aviador / todo así claro y nervioso, / yo te canto, joh jugador maravilloso! / que hoy has puesto el pecho mío como un trémulo tambor!» (1969, p. 3). Los versos que le siguen son, como es de esperar, y reducidos a simples cualidades de Gradín, del mismo tenor vertiginoso: «ágil / fino / alado / eléctrico / repentino / fulminante / yo te vi en la tarde olímpica jugar» (Parra del Riego, 1969, p. 3). ${ }^{2}$

Más allá del potencial representacional de los testimonios estéticos que hemos mencionado, y en los que no procuramos ahondar en esta ocasión, cabe, sin embargo, interrogarse con ánimos exploratorios por una existente contigüidad (no ya exclusivamente historiográfica) entre las condiciones estructurales y culturales de una época y el avance de una práctica como el deporte, ${ }^{3}$ en su versión profesional o de alto rendimiento. Esta sugerente imbricación entre el deporte y la sociedad, advertida no privativamente, aunque sí principalmente, por aquellos que han caído bajo el rótulo de externalistas en los términos de la filosofía del deporte contemporánea, mantiene, sin embargo, sus ambigüedades explicativas. Y es que, a pesar de los numerosos intentos de observadores sociales por desplegar una crítica normativa al deporte de alto rendimiento, al denunciar la perjudicial inmoralidad de los efectos de su práctica (alienación,

2 Una investigación más exhaustiva acerca de la recepción del futurismo italiano en la literatura uruguaya (en la poesía, especialmente), así como de las motivaciones detrás de las loas de Parra del Riego al deporte moderno y de Ferreiro a la aceleración técnica reflejada en los transportes, puede encontrarse en Rocca (2018).

3 Al menos en Uruguay, el fin de siglo supuso la contigüidad histórica entre la institucionalización del deporte y la secularización estatal (Malán, 2020); este proceso, ciertamente, no parece encontrarse demasiado lejos de la utopía tecnofílica, aunque «religiosa-moral», imaginada por Marinetti (2009). 
dopaje, violencia, etcétera), su subordinación a la industria cultural o su anexo con un ambivalente interés de las sociedades contemporáneas sobre el cuerpo, son contadas las ocasiones en las que se reconstruyen los fundamentos y los supuestos que hacen posibles tales críticas. ${ }^{4}$

Los objetivos de este artículo, por consiguiente, son fundamentalmente dos: mostrar la semejanza entre la aceleración, como indicador y valor característico de las sociedades modernas (y tardomodernas), y la lógica social del deporte de alto rendimiento (I), y tomar tal criterio evaluativo como forma de llevar a cabo una posible crítica inmanente al deporte de alto rendimiento, que satisfaga los actuales estándares de la investigación social dedicados a advertir la problemática trascendencia del investigador, al tiempo que sirva como punto de apoyo para futuros estudios respecto de esta normatividad compartida (II). En lo que resta del artículo mantendremos, así, la separación analítica entre ambas coordenadas teóricas, en el afán de evidenciar las causas de sus efectos intuitivamente reprobables, las explicaciones de tales desajustes sociales y, en última instancia, el fondo teórico de los fundamentos normativos que hacen posible una crítica satisfactoria, y no meramente arbitraria, al deporte de alto rendimiento.

Los rasgos del deporte de alto rendimiento o de élite son los que, a primera vista, permiten distinguirlo de otras formas aparentemente más beneficiosas o auténticas, a menudo subsumidas dentro de la categoría de deporte amateur (deporte comunitario, cooperativo, escolar, popular, etcétera), y, a fortiori, imprescindibles para llevar a cabo una crítica a los fundamentos del primero. Podríamos enumerar, sin ánimo de ser exhaustivos, sus principales características como sigue: a) existe una práctica oficial competitiva; b) implica un entrenamiento especializado previo a la competición; c) intervienen profesionales (árbitros, entrenadores, preparadores físicos, nutricionistas, médicos deportólogos, etcétera) para la optimización de la competencia; d) supone una remuneración o una promesa de futura remuneración y seguridad económica y social, y e) comporta una escrupulosa estructura jerárquica (de las divisiones inferiores a las superiores, de los equipos de menor a los de mayor prestigio, de las competiciones locales a las regionales o mundiales, etcétera). plausible al deporte de alto rendimiento, confróntese Bracht (2017). 
La filosofía del deporte, especialmente influyente en la ética del deporte y en la bibliografía especializada de origen anglosajón, ${ }^{5}$ y en su habitual defensa del deporte moderno, ha catalogado como internalistas o interpretacionistas a todas aquellas posiciones que indican que estos principios que hemos enumerado no contienen por sí mismos normatividad alguna, sino que, antes bien, son axiológicamente neutrales con respecto a la vida social, tanto como la especificidad de sus reglas le otorga autonomía a su práctica (Simon, 2000). Esto no significa, sin embargo, que dichas posiciones renieguen de una altamente intuitiva relación entre el deporte y la sociedad, sino que, sencillamente, consideran que es necesario ponderar la búsqueda de la estructura común antes que reparar en sus semejanzas con otras prácticas sociales; de hecho, los análisis hermenéuticos, desde cuyas bases teóricas ha surgido el propio internalismo, siempre se han ocupado de las implicancias morales, políticas y sociales del deporte, aunque más no sea bajo una sintomática indistinción entre el deporte moderno y los juegos deportivos de la Antigüedad (López Frías e Isidori, 2018).

Sin servirnos de las categorías habituales de la teoría social empleadas sobre este fenómeno, e incluso al notar los límites de la propia disciplina de la filosofía del deporte contemporánea, aquí sostendremos que existen elementos comunes entre algunos de los principios enumerados antes, que se suponen fundamentales en lo que respecta a la presunta naturaleza intrínseca del deporte, y la acelerada forma de vida que domina las sociedades contemporáneas. ${ }^{6}$ Emprender una crítica a las formas de vida contemporáneas aparece como una tarea cuando menos arriesgada, si no fútil, para las concepciones habituales del alcance de la filosofía y la teoría social en la actualidad. Sin embargo, recientemente se han podido reconstruir y elucidar sendos modelos alternativos de crítica, en el intento por desligarse de la neutralidad axiológica a la que empuja la plural y vasta tradición del liberalismo político, ${ }^{7}$ que permiten sentar las bases fundamentales para indicar desarrollos sociales malogrados, i.e., formas de vida cuyas consecuencias perversas son por completo indeseadas por los agentes sociales. ${ }^{8}$ Esta indicación de perturbaciones sociales no se ajusta, por lo tanto, a criterios externos o ideales normativos

5 En lo que resta del artículo no emplearemos las categorías habituales de la filosofía del deporte contemporánea, por entender que no son explicativamente satisfactorias como para lidiar con la complejidad del fenómeno social del deporte de alto rendimiento, cuyos dilemas no pueden reducirse causalmente a la fidelidad de un modo de producción y, menos aún, a la corrupción de su asumida naturaleza intrínseca por parte de la vida social. Referirnos al externalismo y al internalismo sirve aquí, más bien, como punto de apoyo a través del cual entrar en diálogo con la bibliografía disponible en la actualidad.

6 Un estudio antecedente sobre la conexión entre la aceleración social y el deporte de alto rendimiento, con énfasis en sus proximidades culturales, puede encontrarse en Vaz (2020).

7 Respecto de la denuncia frente la «privatización» de la interrogación y la formulación normativa acerca de las ideas de vida buena, la ponderación de estilos de vida y los rasgos fundamentales que adquiere cualquier diseño de un plan vital llevado a cabo en sociedades complejas, véase Rosa (1998). 
arbitrariamente escogidos que puedan ser presa de incontables denuncias de perfeccionismo, o incluso de presupuestos filosófico-antropológicos, ${ }^{9}$ sino que brega por ubicar principios inmanentes que se localicen dentro de la propia normatividad del mundo social. Proponernos esto último, desde luego, coloca nuestra tentativa en la estela de la teoría crítica y las distintas generaciones de la Escuela de Frankfurt, ${ }^{10}$ cuyos aportes, sobre todo los más recientes, hemos de atender en lo que sigue del artículo.

La advertencia acerca de la tendencia intrínseca a la aceleración y la lógica escalatoria (logic of escalation) de las estructuras sociales contemporáneas ha sido delineada con precisión por innumerables observadores sociales, aunque tal vez su formulación más acabada se encuentre en Rosa $(2013,2016)$. Este último, conocido actualmente como uno de los representantes de la cuarta generación de la Escuela de Frankfurt, ha logrado detallar las causas, las consecuencias y los principales modos en que se presenta la aceleración social en las sociedades tardomodernas. Además de resultar connatural al proceso de modernización y radicalizarse definitivamente en la modernidad tardía, la aceleración (tecnológica, del cambio social y del ritmo de la vida) contiene factores estructurales y culturales, y no ya solamente internos, que definitivamente contribuyen a propulsarla (Rosa, 2013). Para nosotros, por lo tanto, lo relevante se encuentra en la posibilidad de desarrollar tres aspectos: a) esbozar una posible conexión causal entre la aceleración social y la emergencia del deporte moderno; b) mostrar algunos de los efectos de tal fenómeno social en la competición consustancial al deporte, y c) solventar la neutralidad axiológica con respecto a las concepciones del bien y vida buena que rige la relación o bien profesional, o bien de consumo, que los actores sociales mantienen con el deporte de alto rendimiento en la actualidad. Mientras el primer aspecto será mencionado, aun superficialmente, en lo que resta de esta sección, y el segundo se desarrollará en la sección subsiguiente, el tercero aparecerá como una consecuencia directa o un corolario mayor de nuestro planteo general.

Constituye un verdadero truismo advertir que el deporte moderno no puede comprenderse sin las condiciones de época que han permitido su emergencia; sin embargo, poco se encuentra disponible en la bibliografía especializada acerca de la injerencia dentro del propio funcionamiento social de los principios aparentemente asépticos del alto rendimiento, y no ya de las implicancias sociales y políticas de su ejercicio. Cabe establecer, por lo tanto, que mientras existen deportes que en la actualidad no pueden comprenderse en toda su naturaleza interna sin el peculiar régimen espaciotemporal que aqueja a las

Un ejemplo de esta crítica aplicada al deporte de alto rendimiento puede encontrarse en la actualización del Honneth (2007) de la noción de reificación, y en su ejemplo al respecto de un deportista que olvida la existencia necesaria de su adversario, que también es su amigo, para el propio desarrollo de la competición. 
ciudades contemporáneas, ${ }^{11}$ resulta plausible buscar principios comunes al deporte de alto rendimiento y el fenómeno de la aceleración social moderna y tardomoderna (Vaz, 2020).

No puede ignorarse, de hecho, que la competencia, entendida como el criterio más importante en la actualidad en lo que respecta a la distribución de bienes, recursos y prestigio social (Rosa, 2016), es uno de los elementos basales para el ejercicio deportivo. Como hemos señalado al inicio de esta sección, su propia dimensión profesional, así como su formulación como espectáculo capaz de atraer consumidores de forma masiva, se estructura por entero sobre la garantía de la competición periódica: las incontables críticas sociales desplegadas hacia los megaeventos deportivos, al igual que la condena habitual al mantenimiento diario de las variables biológicas a través de la sucesión inflexible del entrenamiento deportivo, la nutrición y el descanso apropiados, no pueden comprenderse en toda su magnitud sin considerar el rol central que desempeña la competición oficial.

En esta sección podremos llegar a sugerir la tesis de que el deporte, al menos bajo la forma del alto rendimiento, de élite o profesionalizado, está plagado de consecuencias indeseadas, esto es, de desarrollos necesarios que surgen a pesar de los deseos y las motivaciones más profundos de quienes reproducen las condiciones para que ocurra tal ascenso. Estos efectos perversos, comprendidos como un tipo de consecuencias indeseadas, denotan cierto estado paradójico de cosas en el que las expectativas de un agente, aunque auténticas, no concuerdan con las intenciones originales y se presentan por completo heterónomas frente a estas últimas (Giddens, 1987). Casos de consecuencias no intencionales, por ejemplo, pueden identificarse en los embotellamientos recurrentes en las ciudades contemporáneas (Rosa, 2016), en la depresión patológica como efecto del ansia de intensidad permanente (Garcia, 2018), o incluso en la denuncia del mundo académico acerca de la falta de tiempo para dedicar a la investigación, la enseñanza o cualquier otra tarea que sobrepase la gestión institucional (Noonan, 2015).

En lo que respecta al deporte de alto rendimiento, en el que el principio de competencia regula tanto el ejercicio oficial como la propia dinámica inherente a su ambiente acelerado, escalar y autotélico, ${ }^{12}$ una de las consecuencias indeseadas tal vez se

11 La emergencia y la consolidación de deportes como el parkour, por ejemplo, que no necesariamente son de élite o buscan su profesionalización, solo puede comprenderse a la luz de la aceleración social; su originalidad, de hecho, es sintomática de una forma particular de habitar las ciudades contemporáneas y la peculiar contracción del tiempo presente que por norma general las aqueja (Højbjerre, 2021). 
concrete en la difusión a escala global, e incluso en ámbitos que se desenvuelven en ausencia de competiciones oficiales (Sánchez Leyva, 2019), del empleo recurrente de sustancias ilícitas, que alteran la habitual capacidad de regeneración biológica. De hecho, los incontables criterios de corrección legal y moral que hoy existen y son divulgados públicamente con frecuencia parecen resultar sintomáticos de la importancia que el tema ha cobrado dentro de los estudios dedicados a las implicancias sociales o sociopolíticas del deporte de alto rendimiento, al igual que de la desesperación que los organismos locales e internacionales mantienen a causa de la relativa imposibilidad de limitar su utilización. El imperativo del dopaje, que en ocasiones parece ser más nuclear de la propia lógica social del deporte de alto rendimiento que una efectiva subversión de su presunta naturaleza incorrupta (Missa, 2015), pone en evidencia la presión que empuja, estructuralmente, a los deportistas a la búsqueda de ventajas ilegítimas (Garcia, 2018). ${ }^{13}$ Evidentemente, para concebir al dopaje (doping) o a la «mejora humana» en general, como un efecto necesario, aunque excepcionalmente deseado, para ingresar en la competencia tout court dentro del ámbito del deporte de alto rendimiento, debemos asumir la tesis de que los deportistas profesionales no se sirven de ayudas ergogénicas ilícitas tan solo por motivación o incluso como forma de ejercer su autonomía personal; ${ }^{14}$ lo que puede suceder, por el contrario, es que las estructuras sociales contribuyan a la reproducción y el mantenimiento, pero también al cambio, de ciertas dinámicas sociales perversas y reprobables según nuestras más difundidas intuiciones morales. El dopaje, por lo tanto, no sería satisfactoriamente explicado al apuntar su causa a las escasas virtudes éticas y cívicas que logran desarrollar la mayoría de los deportistas profesionales, sino al poner de manifiesto la imposibilidad de estos últimos de desligarse del vértigo social que domina todas las esferas que atañen a su realización personal. La absolutización de la jornada por parte del entrenamiento y la regeneración, que necesariamente deben ser periódicos y

dida como beneficiosa para promover la motivación intrínseca, determinante en la adherencia a la actividad física o incluso al propio ejercicio deportivo (Mikicin, 2013) e integrada en la célebre definición del deporte moderno por parte de Guttmann (2004a). Por el contrario, aquí sugerimos que, menos que autotélicas, las implicancias de la competición deportiva suelen ser por completo heterónomas para sus participantes.

13 Desde esta perspectiva quedarían de lado, al menos para agotar el fenómeno del dopaje, aquellas explicaciones metodológicamente individuales que ponderan los desajustes reflexivos o el desarrollo poco autónomo de la agencia moral y, en consecuencia, advierten la necesidad de que los deportistas profesionales adopten conductas moralmente ejemplares, excelencia atlética y virtudes cívicas.

14 Una de las formas en que se ha procurado defender la posibilidad de que los deportistas decidan libremente si deben recurrir a ayudas ergogénicas ilegales para mejorar su rendimiento ha sido, de hecho, la de la autonomía moral y ética (López Frías, 2015). Con todo, es justo decir que, al menos para la teoría crítica de la sociedad, el problema es, y ha sido desde siempre, lo que yace detrás de la autonomía: son las afectaciones a sus condiciones de posibilidad lo que generalmente se ha denunciado bajo la idea del consumismo, la manipulación de las necesidades o, entendida como verdadera patología de lo social, la alienación. 
sostenerse en el tiempo para llegar a ser exitosos, al igual que por la competición oficial que les sirve de horizonte, son los ejemplos más prístinos en los que se exponen las consecuencias de tales desajustes sociales (Vaz, 1999). ${ }^{15}$

Como uno de los modos en los que se presentan los efectos paradójicos y no intencionales del deporte, que en este caso afecta directamente a los deportistas profesionales (o no profesionales) involucrados, el dopaje constituye un caso cuando menos interesante, pues en él se ponen de manifiesto determinaciones externas tanto como motivaciones internas, i.e., apropiadas y reproducidas por los propios agentes. De ahí, precisamente, que optemos por desembarazarnos de las categorías con las que trabaja la filosofía del deporte actual, aunque advirtamos su utilidad al distinguir entre esferas y sus respectivas especificidades en cuanto a la reproducción y modificación de cierto estado de cosas general. Las causas del dopaje no parecen responder exclusivamente a desajustes psicológicos individuales, ciertamente, pero tampoco a una reproducción autopoiética de estructuras sociales opresoras. ${ }^{16}$ Menos aún, incluso, deben ser identificadas como atentados contra la presunta naturaleza originaria del deporte, que por lo general no hace sino impulsar tal empleo de sustancias.

Por el contrario, nuestra tesis fundamental en este punto es que el deporte de alto rendimiento depende de la reproducción de estructuras sociales ontológicamente más amplias al igual que contribuye a ella, al tiempo que conecta con aspiraciones, demandas y motivaciones, muchas de las cuales se engloban en cierta concepción de vida buena, que coadyuvan a la consagración social de aquel en el mundo contemporáneo. Por ello, podríamos establecer que la habitual tendencia maniquea a identificar al deporte de alto rendimiento, o bien como un síntoma transparente o un espejo de la forma de vida capitalista, o bien como un territorio autónomo amenazado en su presunta naturaleza intrínseca, no parece suficiente para lidiar con la complejidad del fenómeno social que se adosa en tal práctica. Con esto no hacemos referencia tan solo a los diversos procesos de apropiación y resignificación en el entorno local de los grandes espectáculos deportivos, sino, más aún, a aquellas motivaciones internas que se acoplan con las promesas, sean estas profesionales o de consumo, bajo las que se modula el deporte de alto rendimiento. Las promesas, de hecho, son formas secularizadas, o incluso «equivalentes funcionales» según las que los actores sociales orientan su existencia, al necesariamente

15 Esto se observa con suficiente precisión, también, en los incontables estudios empíricos en los que se documentan las dificultades que los deportistas profesionales jóvenes mantienen para conciliar el tiempo periódicamente dedicado al entrenamiento (incluida su recuperación) con las habituales exigencias educativas formales (Mateu, 2021).

Si bien Guttmann (2004b), en su crítica a las visiones externalistas sobre el fenómeno deportivo, advierte la función primordial que cumplieron las competiciones en los regímenes comunistas, no considera, desde luego debido su publicación en 1978, las implicaciones de la caída del socialismo real. 
tener que lidiar con la conciencia de finitud y ya no con la esperanza de trascendencia o vida eterna promulgada por la tradición cristiana (Rosa, 2013, 2016). ${ }^{17}$

La mayoría de las promesas culturales que, aun secularizadas, envuelven al deporte de alto rendimiento, por norma general se mantienen incumplidas. Para el joven deportista o atleta de élite, por ejemplo, ya sea que su sueño de alcanzar la profesionalidad (y la consecuente seguridad económica y social que le sigue) se confunda con el resto de aquellos que aspiran exactamente al mismo objetivo, o incluso al verse obligado a incurrir en atajos para aventajar su rendimiento frente al resto de los competidores (indistintos al carácter de rivales deportivos, compañeros de equipo, colegas u otras jóvenes promesas), las limitaciones de su más auténtico anhelo se manifiestan rápidamente. Mientras tanto, al consumidor periódico de los eventos deportivos basta interrogarlo por lo que pasaría si dejara de atender a las vicisitudes de su equipo favorito durante no más de dos o tres años; probablemente, en este último caso, dejaría de conocer a la mayoría de los integrantes de la plantilla, los profesionales que intervienen en la optimización de la competencia oficial, las transferencias, los rankings, récords y resultados más salientes, ${ }^{18}$ y cualquier otra información en la que creía observar una señal o principio lo suficientemente sistemático como para gestar un vínculo afectivo y prolongado.

Advertir esta última dimensión cultural en la que desembocan tanto el deporte de alto rendimiento como el vértigo social que se propulsa desde las estructuras sociales contemporáneas nos permite entrever las virtudes que contiene una crítica, no ya estrictamente moral, como en el caso del dopaje, al punto de encuentro entre ambos dominios. ${ }^{19}$ Por ello, las críticas normativas al consumo de los espectáculos deportivos, o incluso a sus megaeventos periódicos, exigen explicaciones filosófica y sociológicamente satisfactorias, que no pueden reducirse a la documentación de efectos en desajustes reflexivos o inhibición de la capacidad de autonomía. Por el contrario, la etiología compartida entre todos aquellos fenómenos de consumo masivo, usualmente transmitidos por medios audiovisuales digitales (al menos en lo que respecta a las competiciones internacionales), también exhibe la experiencia compartida en cuanto a la insatisfacción posterior a su consumo: al igual que la atracción del consumo televisivo habitual no puede explicarse sin la relación costo-beneficio que le subyace (Rosa, 2013,

17 No es casual que, en lenguaje coloquial, y al menos en el español rioplatense, el epíteto promesa les sirva a los hablantes para significar a aquellos jóvenes deportistas, destacados por su rendimiento, en los que se proyectan trayectorias futuras de realización personal, prestigio y reconocimiento nacional e internacional.

18 La cuantificación concretada en escalas, rankings, récords, así como la documentación de prácticamente todos los resultados obtenidos durante una competición oficial constituyen, al decir de Guttmann (2004b), la cristalización de la idea misma de deporte moderno.

19 Sobre las diferencias constatables entre una crítica moral y una crítica ética para la vida social contemporánea, véase Rosa (2016). 
2016), tampoco la consagración social del deporte de alto rendimiento parece poder explicarse sin considerar la promesa de intensidad que subyace, o bien a su producción, o bien a su consumo (Garcia, 2018), pero que en ningún caso parece satisfacerse.

La hipótesis de análisis filosófico-social, y hasta propiamente sociológico, que hemos podido esbozar hasta aquí conduce a concluir que la difusión del dopaje sucede tanto porque el régimen espaciotemporal de las sociedades contemporáneas impide la completa desaceleración (o, en su defecto, porque sus costos aparecen como demasiado elevados) como porque los actores sociales, i.e., sus profesionales y sus consumidores, creen encontrar algún elemento de autorrealización, bienestar o satisfacción personal en el sostenimiento periódico de su consagración social.

Por lo tanto, puede afirmarse que el empleo de sustancias para lograr ventaja deportiva ante los posibles adversarios, i.e., potencialmente todos los actores sociales, tiene lugar porque los deportistas profesionales no pueden cesar el conflicto prometeico que los aqueja, y al cual deben contribuir a reproducir cíclica e inacabadamente a través de un entrenamiento sistemático (Vaz, 1999) que, desde luego, se halla al amparo de una concepción progresiva, lineal e infinita del tiempo (Vaz, 2020). ${ }^{20}$ Asimismo, debido a que las motivaciones intrínsecas (autorrealización, satisfacción y superación personal, gratificación por la adquisición de nuevas habilidades, etcétera), pero sobre todo extrínsecas (ansia de reconocimiento y prestigio social, acceso a bienes y recursos únicamente disponibles a los más aventajados, etcétera), ${ }^{21}$ inevitablemente se encuentran presentes en las aspiraciones de quienes anhelan dedicarse profesionalmente al deporte de alto rendimiento (Coupland, 2015). El objetivo analítico y metodológico principal de este artículo ha sido, según lo visto hasta aquí, evidenciar que, mientras la primera dimensión explicativa excede a la crítica moral y al correspondiente vocabulario que la filosofía práctica del deporte emplea por norma general, la segunda puede satisfacerse con explicaciones individuales y no necesariamente socioestructurales ni obligatoriamente culturales.

Si bien Adorno (1998) lamentaba la racionalización excesiva que acompaña a la jornada del deportista profesional, ${ }^{22}$ junto con la consecuente desafección y rigidez que provoca la obligada administración del dolor en el entrenamiento, la competición y su

20 Rosa (2013) ha elucidado, en el marco de la reconstrucción de su original teoría de la aceleración social, la compatibilidad, y no así relación de exclusión, entre la temporalidad circular y la lineal dentro de las estructuras sociales contemporáneas.

21 Acerca de la distinción entre motivaciones intrínsecas y motivaciones extrínsecas, y su rol en la explicación de las razones de la participación voluntaria de jóvenes en las competiciones deportivas, véase van Wersch (1997).

22 Al contrario de Adorno, Rosa (2020) cree ver en el deporte no solo una oportunidad de racionalización y prognosis, sino también de apertura e incontrolabilidad; con todo, este juicio solo parece ser posible bajo una problemática coextensividad entre deporte y juego, no necesariamente compartida por las generaciones antecedentes de la teoría crítica. 
repetición cíclica, ${ }^{23}$ cabe preguntarse si estas son las únicas consecuencias posibles de la consagración social del deporte y, más aún, de su voluntaria elección profesional. Asimismo, y tal vez con mayor pertinencia para el devenir de la filosofía y la teoría social, conviene interrogarse por el carácter explicativo de tales coordenadas, ancladas en la intuitiva conexión entre el capitalismo contemporáneo y la promoción generalizada de la racionalidad instrumental. Así, es probable que el reconocimiento de la coexistencia de elementos causales, rastreables tanto en las estructuras sociales como en motivaciones intrínsecas, inevitablemente subordinadas a deseos de autorrealización auténtica, permita constituir una heurística más satisfactoria al momento de explicar la gran difusión de las formas deportivas de rendimiento dentro de las sociedades contemporáneas, que no necesariamente resulta captada por la advertencia del exceso de racionalización.

\section{Consideraciones finales}

En este trabajo nos hemos servido de algunos desarrollos contemporáneos en teoría y filosofía social para poner de relieve las semejanzas intrínsecas entre la aceleración social de la modernidad tardía y el deporte de alto rendimiento. Hemos sugerido que el vértigo social que aqueja a las sociedades contemporáneas puede hallarse, también, en las exigencias que los deportistas profesionales deben satisfacer para no perder su ventaja competitiva frente al resto de los potenciales adversarios. Asimismo, los profesionales del deporte de élite suelen complementar estas exigencias, usualmente heterónomas, como se observa en el caso del dopaje, con auténticos deseos de autorrealización, superación y satisfacción personal. Así, además de intentar una superación de las categorías habituales que emplea la filosofía del deporte contemporánea, procuramos considerar factores o mecanismos culturales que, como en el caso de la promesa secularizada de vida eterna, las consecuencias indeseadas y los efectos paradójicos de su ejercicio, intentan explicar la causa de su consagración social, tanto en su producción como en el consumo masivo de su ejercicio. Finalmente, la funcionalidad que contiene el deporte de alto rendimiento en la reproducción de cierto estado de cosas general, i.e., de la forma de vida capitalista, debido a su dependencia de la lógica escalatoria imbricada con la aceleración social, desafían algunas de las explicaciones sociales contemporáneas y advierten la complejidad inherente a la relación deporte-sociedad que se entreteje en la actualidad.

El abandono voluntario a la experiencia del dolor, que parece ser fundamental para el análisis social del deporte de alto rendimiento, e incluso necesario para explicar la naturaleza del dopaje, no puede comprenderse, ciertamente, sin el fondo teórico secularizado que ha coadyuvado a su realización dentro de las sociedades contemporáneas. 


\section{Referencias bibliográficas}

Adorno, T. (1998). Educación para la emancipación. Madrid: Morata.

Bracht, V. (2017). Esboço de uma crítica imanente do esporte de alto rendimento. Ponencia presentada en el $12 .{ }^{\circ}$ Congreso Argentino y 7. ${ }^{\circ}$ Latinoamericano de Educación Física y Ciencias. UNLP, Argentina. Recuperado de http://www.memoria.fahce.unlp.edu.ar/trab_eventos/ev.10106/ev.10106.pdf.

Coquio, C. (2021). Aceleraciones: Una introducción. Cuadernos LÍRICO, 22, 1-17. Recuperado de https://journals.openedition.org/lirico/pdf/10834.

Coupland, C. (2015). Entry and exit as embodied career choice in professional sport. Journal of Vocational Behavior, 90, 111-121. doi: 10.1016/j.jvb.2015.08.003.

Fleck, A. (2017). Afinal de contas, o que é teoria crítica? Princípios: Revista de Filosofia, 24(44), 97-127. doi: 10.21680/1983-2109.2017v24n44ID12083.

Garcia, T. (2018). The life intense: A modern obsession. Edimburgo: Edinburgh University Press.

Giddens, A. (1987). Social theory and modern sociology. Stanford: Stanford University Press.

Guttmann, A. (2004a). Sports: The first five millennia. Amherst: University of Massachusetts Press.

Guttmann, A. (2004b). From ritual to record: The nature of modern sports. Nueva York: Columbia University Press.

Højbjerre, S. (2021). «Parkour: Playing the modern, accelerated city. Journal of the Philosophy of Sport, 48(1), 26-44. doi: 10.1080/00948705.2020.1834862.

Honneth, A. (2007). Reificación: Un estudio en la teoría del reconocimiento. Buenos Aires: Katz.

Ialongo, E. (2013). Filippo Tommaso Marinetti: The futurist as fascist, 1929-37. Journal of Modern Italian Studies, 18(4), 393-418. doi: 10.1080/1354571X.2013.810800.

López Frías, F. (2015). Mejora humana y dopaje: Una propuesta crítica. Madrid: Reus.

López Frías, F., e Isidori, E. (2018). Los orígenes deportivos de la democracia: Una aproximación filosófica. Movimento, 24(2), 495-508. DOI: 10.22456/19828918.78461.

Malán, P. (2020). Deportivización y proceso de secularización en el Uruguay (18701930). Revista Brasileira de Educação Física e Esporte, 34, 39-49. Recuperado de https://www.revistas.usp.br/rbefe/article/view/173143/162480.

Marinetti, F. T. (2009). The new religion-morality of speed. En H. Rosa y W. E. Scheuerman (eds.), High-speed society: Social acceleration, power, and modernity (pp. $57-$ 59). Pennsylvania: Pennsylvania State University Press.

Mateu, P., Vilanova, A., Torregrossa, M., y Inglés, E. (2021). Estudiar y competir en las sociedades aceleradas ¿Una carrera a tumba abierta?. The Journal of the Latin 
American Socio-Cultural Studies of Sport, 13(1), 133-149. Recuperado de https://revistas.ufpr.br/alesde/article/view/ 77597/43757.

Mikicin, M. (2013). Autotelic personality as a predictor of engagement in sports. Biomedical Human Kinetics, 5, 84-92. doi: 10.2478/bhk-2013-0013.

Missa, J.-N. (2015). El deporte de competición, laboratorio de la medicina del mejoramiento: Análisis ético y filosófico del problema del dopaje. Revista Colombiana de Bioética, 10(2), 210-226. Recuperado de https://revistas.unbosque.edu.co/index.php/RCB/article/view/1766/1348.

Noonan, J. (2015). Thought-time, money-time, and the temporal conditions of academic freedom. Time \& Society, 24(1), 109-128. doi: 10.1177/ $0961463 X 14539579$.

Parra del Riego, J. (1969). Polirritmos. Montevideo: Arca.

Rocca, P. (2018). Uruguay. En G. Berghaus (ed.), Handbook of international futurism (pp. 871-882). Berlín: De Gruyter. doi: 10.1515/9783110273564.

Rosa, H. (1998). On defining the good life: Liberal freedom and capital necessity. Constellations, 5(2), 201-214. doi: 10.1111/1467-8675.00088.

Rosa, H. (2013). Social acceleration: A new theory of modernity. Nueva York: Columbia University Press.

Rosa, H. (2016). Alienación y aceleración: Hacia una teoría crítica de la temporalidad en la modernidad tardía. Buenos Aires: Katz.

Rosa, H. (2020). The uncontrollability of the world. Cambridge: Polity Press.

Sánchez Leyva, M. (2019). Los mitos de la salud y el deporte: La elección del dolor como ofrenda neoliberal. La Torre del Virrey, 1(25), 171-179. Recuperado de https://revista.latorredelvirrey.es/LTV/article/view/108/87.

Simon, R. (2000). Internalism and internal values in sport. Journal of the Philosophy of Sport, 27, 1-16. doi: 10.1080/00948705.2000.9714586.

van Wersch, A. (1997). Individual differences and intrinsic motivations for sport participation. En J. Kremer, K. Trew y S. Ogle (eds.), Young people's involvement in sport (pp. 53-72). Londres: Routledge.

Vaz, A. (1999). Treinar o corpo, dominar a natureza: Notas para uma análise do esporte com base no treinamento corporal. Cadernos Cedes, 19(48), 89-108. Recuperado de https://www.scielo.br/j/ccedes/a/d6WVcPMpmPBCLhrfdmSGHkQ/?lang= pt\&format $=$ pdf.

Vaz, A. (2020). Pesquisar esportes em humanidades: Abordagens, temas, possíveis ideias. Novos Olhares Sociais, 3(1), 111-126. Recuperado de https://www3.ufrb. edu.br/ojs/index.php/novosolharessociais/article/view/502/240. 